\title{
Performing safety functions to monitor the protected area using a light curtain
}

\author{
Juraj Ždánsky, Milan Medvedík \\ Department of Control and Information Systems \\ University of Žilina, Faculty of Electrical Engineering and Information Technology \\ Žilina, Slovakia \\ juraj.zdansky@fel.uniza.sk, milan.medvedik@fel.uniza.sk
}

\begin{abstract}
In many applications, a light curtain is used to detect the intrusion of a human (or other protected object) into the danger zone. After detecting the interruption of the light curtain, a safety function is activated to bring the danger zone into a safe state (nonhuman). If the space taken by the light curtain is at the same time a working space, it is necessary to distinguish whether the light curtain interrupt is caused by man or material (material flows cannot cause the dangerous zone to be put into a safe state). There are several ways how to achieve this resolution, but additional (optional) sensors are commonly used. The paper deals with the parameters of additional sensors and how to connect them to the achieved Safety Integrity Level (SIL) of the safety functions realized by the help of the light curtain.
\end{abstract}

Keywords - safety light curtain; SIL; probability; CTMC

\section{INTRODUCTION}

One of the most common means of detecting the presence of an object is a light curtain (LC). Its usage can be found in a variety of applications ranging from consumer electronics to safety applications. Just such an application is dedicated to this post. These are applications that, in addition to the required operation, also require the achievement of the required Safety Integrity Level (SIL) in accordance with the requirements of EN IEC 61508 [1]. Currently, such applications are not used only in transport but also in industry. A common feature of safety features utilizing a light curtain is the secure detection of the object and the subsequent reaction, usually putting the dangerous zone in the safe state behind the light curtain. The situation is illustrated in Fig. 1. The danger zone poses a danger to the moving object therefore the light curtain is in distance $l$ from the danger zone ([2] deals with the determination of this distance in detail deals with the determination of this distance in detail). Its purpose is to detect object input. Information about the interruption of the light curtain is processed by the control system (e.g. safety PLC (Programmable Logic Controller)); the safety PLC is comprehensive control system that can perform complicated standard functions simultaneously with performing safety functions, [3]), which subsequently (according to the algorithm implemented in the control software) influences the hazard generating elements in a way that the danger disappears.

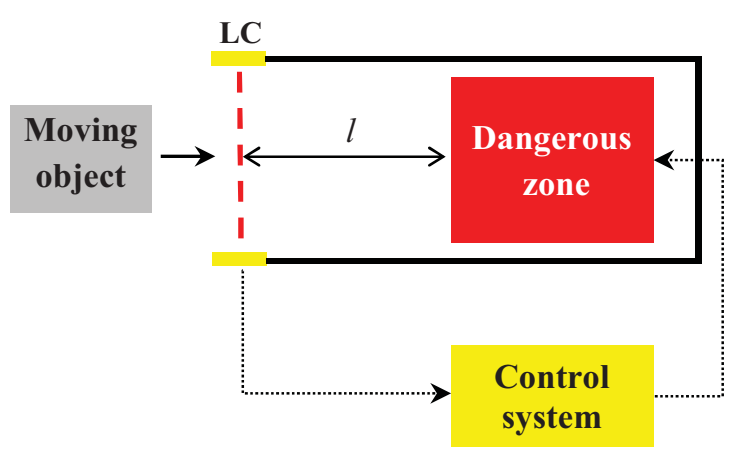

Fig. 1. Protection of the moving object before entering the danger zone.

If the hazard generated by a dangerous zone can potentially cause a threat to the health or life of the person, damage to the environment, or large material damage, then the function depicted in Fig. 1 is called the safety function. It eliminates potential hazards. These must be eliminated with SIL based on risk analysis. The use of certified software blocks and the subsequent verification of the application guarantees the systematic integrity of the safety function of the implemented safety function (for example, see [4], [5], [6]). The integrity of the safety against accidental failures is determined by the components used and the way they are connected. In order to determine the achieved SIL for specific components, their involvement, parameters and communication links, a suitable model should be developed. This issue is addressed by many publications [7], [8], [9], [10], [11], [12].

For the rate of dangerous safety function failure realized according to Fig. 1 applies:

$$
\lambda_{S F}^{D}=\lambda_{L C}^{D}+\lambda_{C S}^{D}+\lambda_{A}^{D}
$$

where $\lambda_{L C}^{D}$ is the rate of dangerous failure light curtain, $\lambda_{C S}^{D}$ is the rate of dangerous control system failure and $\lambda_{A}^{D}$ is the rate of dangerous actuator failure.

Probability of dangerous safety function failure is expressed by equation:

$$
\begin{gathered}
P_{S F}^{D}(t)=1-\left(1-P_{L C}^{D}(t)\right)\left(1-P_{C S}^{D}(t)\right) . \\
\cdot\left(1-P_{A}^{D}(t)\right),
\end{gathered}
$$

where $P_{\mathrm{LC}}^{D}(\mathrm{t})$ is the probability of dangerous light curtain failure, $P_{\mathrm{CS}}^{D}(\mathrm{t})$ is the probability of dangerous 
control system failure and $P_{\mathrm{A}}^{D}(\mathrm{t})$ is the probability of dangerous actuator failure.

Assuming the exponential distribution of failures probability (generally acceptable assumptions for electronic devices), the following applies:

$$
P_{S F}^{D}(t)=1-e^{-\lambda_{S F}^{D} \cdot t} .
$$

\section{THE MOST FREQUENT USE OF LIGHT CURTAIN}

In Fig. 1 is the basic case of using the light curtain. The light curtain used for this purpose must meet precisely defined properties. It is not just a simple optical system (transmitter-receiver), but a whole optical system with internal logic of evaluation. The design of this optical system is mentioned by [13]. The properties of this system (e.g. the number of beams used for object detection, their minimum distances, etc.) define the relevant standards according to the purpose of the use of the light curtain [14].

The implementation of some safety functions however requires not only the detection of the passage of an object in a certain location but also species determination of object entering the dangerous zone or to determine the direction through which the object passes through the light curtain. Then for a defined object type, for some direction of movement through the light curtain, interruption of the light curtain will not cause the dangerous zone to be brought into a safe state (this object or direction is allowed) and vice versa for another object or the direction of the movement indicates that the dangerous zone is in a safe state (this object or direction is forbidden). The most common is to distinguish the entry of a person into the danger zone from the entrance of a technical device (e.g. a vehicle). Such resolution can be done by using additional sensors. Incorrect evaluation of the object, resp. the direction of movement would lead to a dangerous situation, such a solution should be subjected to a safety analysis.

\section{A. Implementing safety functions that determine the type of object}

Fig. 2 shows the most common cases of placing additional sensors enabling to distinguish the object type moving through the light curtain. The object-type resolution principle is to evaluate the sequence of the individual sensor readings. When moving the object as shown in the figure, the pair of sensors S1 and S2 is first affected by, then the light curtain is interrupted, and then the pair of sensors S3 and S4 is affected by. The logic evaluating this sequence subsequently expects the successive releases of the S1 and S2 sensors, then releasing the light curtain and finally releasing the pair of sensors S3 and S4. By following this sequence of influencing and releasing the individual sensors, the moving object is evaluated as an object that has access to the dangerous zone enabled and the MUTING function is activated (to suppress the interruption of the light curtain). As a result, the dangerous zone is not brought into a safe state. The location of the sensors and the type of sensors must be such that an object that does not have access to a dangerous zone (mostly a human) does not cause a defined sequence of influence and release of sensors S1, S2, S3 and S4. Light curtain interruption without affecting the sensors will cause the dangerous zone to be brought into a safe state.

Fig. 2a) represents the case where inductive sensors are used. In Fig. 2b) optical sensors are shown. In both cases, it is necessary to choose their geometric arrangement so that the objects are safely distinguished (especially geometric dimensions and object material should be considered). Fig. 2c) illustrates the use of two crossover optical sensors. Their appropriate location makes it possible to detect the object both before and behind the light curtain.

The evaluation of information from individual sensors and the subsequent MUTING function can be performed either directly in the firmware of the light curtain or in the application software of the control system (depending on the type of light curtain used).

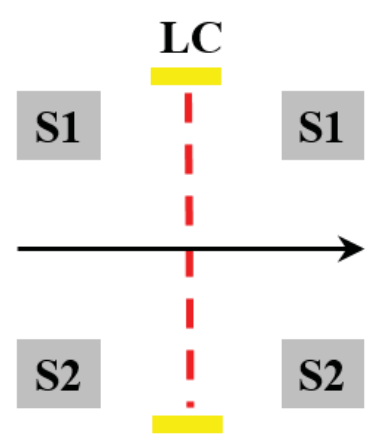

a)

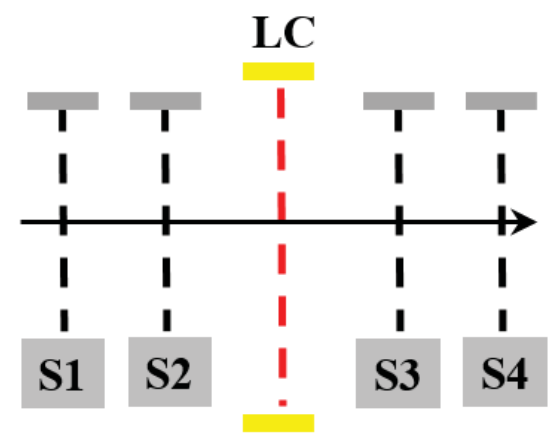

b)

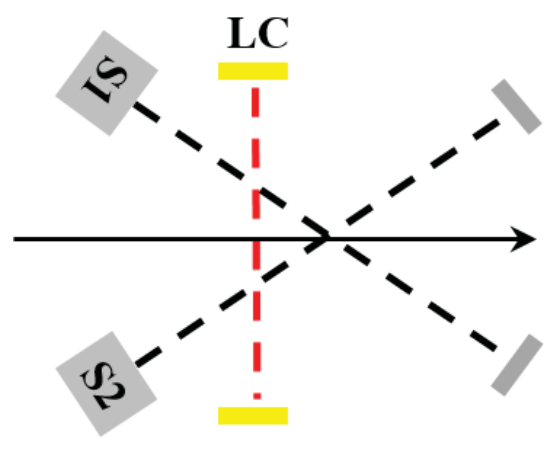

c)

Fig. 2. The most common cases of sensor placement to distinguish an object type moving through a light curtain 


\section{B. Implementing safety functions that distinguish the object's direction}

Fig. 3 shows the case of using a light curtain with detection of the motion direction (the direction of movement shown in the figure is a direction allowed the MUTING function is activated). Even in this case, additional sensors are used. Fig. 3a) shows the use of inductive sensors and Fig. 3b) shows the use of optical sensors. Movement of the object from the dangerous zone is allowed and when the light curtain is interrupted, the MUTING function is activated and the movement of the object towards the dangerous zone is not allowed and the MUTING function is not activated.

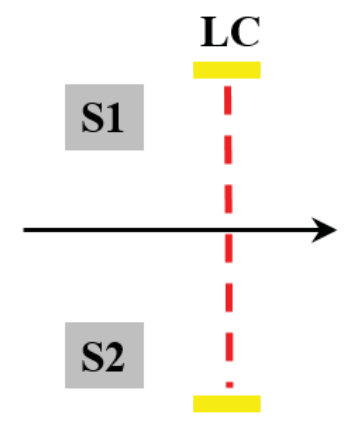

a)

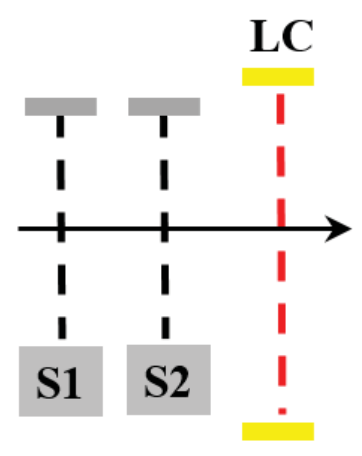

b)

Fig. 3. Light curtain with motion detection

\section{ANALYSIS OF LIGHT CURTAIN SAFETY}

If the light curtain is one of the hardware components performing the safety function, it is necessary to deal with its safety. In the simplest case, a light curtain can be used without additional sensors (without MUTING function, Fig. 1.). In this case, the data from the manufacturer of the light curtain is directly usable in analyzing the safety of the implemented safety function. If the light curtain has a MUTING function, it is also necessary to address the effects of additional MUTING sensors for safety.

\section{A. Light curtain without MUTING function}

In case without MUTING function of light curtain, a failure can be defined as a failure to declare a light curtain if an object is located in its detection zone. In Fig. 4 there is a Continuous Time Markov Chain (CTMC) describing the transition without MUTING function of light curtain from a safety state (state 1) to a dangerous state (state 2 ).

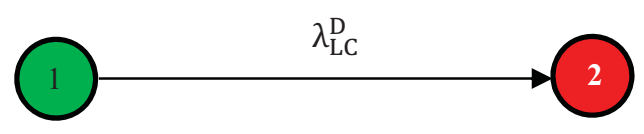

Fig. 4. CTMC - the occurence of a failure LC without MUTING function

From the CTMC in Fig. 4 a relation for probability dangerous failure calculation of the light curtain can be derived:

$$
\mathrm{P}_{\mathrm{LC}}^{\mathrm{D}}(\mathrm{t})=1-\mathrm{e}^{-\lambda_{\mathrm{LC} \cdot}^{\mathrm{D}} \cdot \mathrm{t}}
$$

\section{B. Light curtain with MUTING function}

The light curtain with MUTING function may define a failure as a failure to declare the light curtain in the case when there is an unauthorized object in its detection zone, or a failure to signal the light curtain interruption if the object passes through the detection zone in the unauthorized direction. In this case, a dangerous failure may result in a failure of the light curtain, or the failure of the additional sensors to provide the object type or its direction.

The occurrence of a failure of a MUTING function with light curtain may be described by the CTMC in Fig. 5. The CTMC is assembled on condition that the additional sensors providing the object type or its direction are identical $\left(\lambda_{S}=\lambda_{S 1}=\lambda_{S 2}\right)$.

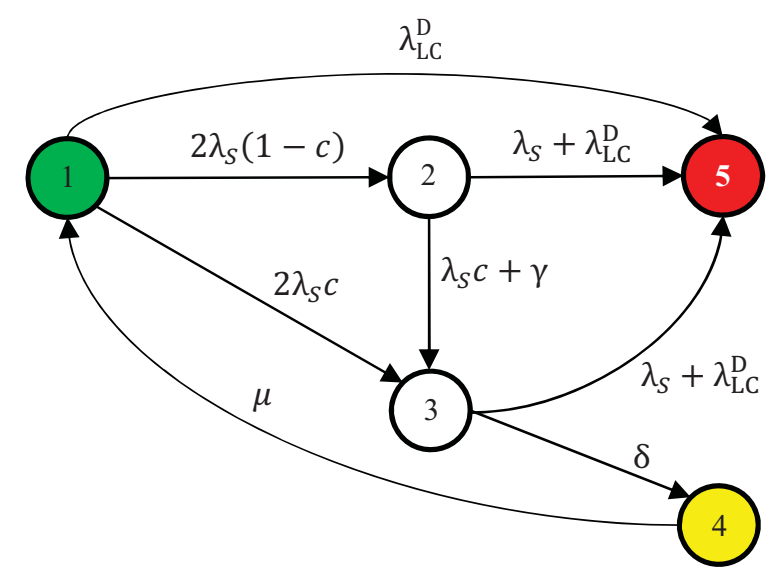

Fig. 5. CTMC - the occurrence of a failure LC with MUTING function

From state 1 (without failure state), LC with MUTING function can reach:

- state 5 (dangerous state) after a dangerous failure of the light curtain;

- $\quad$ state 2 after occurrence of undetectable sensor error $\mathrm{S} 1$ or $\mathrm{S} 2$ ( $c$ is the coefficient of diagnostic coverage);

- $\quad$ state 3 after occurrence of detectable sensor failure $\mathrm{S} 1$ or $\mathrm{S} 2$.

From state 2, LC with MUTING function can go to:

- the state 5 (dangerous state) after the occurrence of a dangerous failure of the light 
curtain or after a failure of sensor S1 or S2 (both sensors have a failure);

- $\quad$ state 3 after occurrence of detectable sensor failure S1 or S2 (this is the occurrence of a detectable failure of the same sensor on which an undetectable failure has already occurred) or after the sensor is affected by the object (if the sensor is in a state of undetectable failure and after the sensor is affected by the object this failure becomes detectable).

From state 3, LC with MUTING function can go to:

- the state 5 (dangerous state) after the occurrence of a dangerous failure of the light curtain or after a failure of sensor S1 or S2 (both sensors have a failure);

- $\quad$ state 4 (safe state after failure detection) after a discrepancy between information from $\mathrm{S} 1$ and S2 sensors lasting longer than a defined time interval.

From state 4, LC with MUTING function can go to state 1 after system restoration. The rate of this transition is

$$
\mu=\frac{1}{\mathrm{MDT}}
$$

where $M D T$ is mean down time.

The rate of the transition from state 2 to state 3 after the sensor is affected by the object depends on how often the object passes through the sensor. Since it does not have to be a regular interval, we will start from a pessimistic assumption and consider the longest interval between two consecutive objects $t_{\max }$. Then:

$$
\gamma=\frac{1}{t_{\max }}
$$

The rate of transition from state 3 to state 4 is rated by:

$$
\delta=\frac{1}{\mathrm{t}_{\text {disc }}}
$$

where $t_{\text {disc }}$ is discrepancy time of sensors S1 and S2.

CTMC in Fig. 4 does not consider sensor S3 and S4 failures (the cases shown in Fig. 2a) and 2b)) because these sensors do not affect the occurrence of a dangerous state (transition to state 5).

CTMC in Fig. 4 can be described as a system of differential equations

$$
\begin{aligned}
\mathrm{P}_{1}^{\prime}(\mathrm{t})= & -\left(\lambda_{\mathrm{LC}}^{\mathrm{D}}+2 \lambda_{\mathrm{S}}(1-\mathrm{c})+2 \lambda_{\mathrm{S}} \mathrm{c}\right) \cdot \mathrm{P}_{1}(\mathrm{t})+ \\
& +\mu \cdot \mathrm{P}_{4}(\mathrm{t}), \\
\mathrm{P}_{2}^{\prime}(\mathrm{t})= & 2 \lambda_{\mathrm{S}}(1-\mathrm{c}) \cdot \mathrm{P}_{1}(\mathrm{t})-\left(\lambda_{\mathrm{S}}+\lambda_{\mathrm{LC}}^{\mathrm{D}}+\right. \\
& \left.+\lambda_{\mathrm{S}} \mathrm{c}+\gamma\right) \cdot \mathrm{P}_{2}(\mathrm{t}), \\
\mathrm{P}_{3}^{\prime}(\mathrm{t})= & 2 \lambda_{\mathrm{S}} \mathrm{c} \cdot \mathrm{P}_{1}(\mathrm{t})+\left(\lambda_{\mathrm{S}} \mathrm{c}+\gamma\right) \cdot \mathrm{P}_{2}(\mathrm{t})-\left(\lambda_{\mathrm{S}}+\right. \\
& \left.+\lambda_{\mathrm{LC}}^{\mathrm{D}}+\delta\right) \cdot \mathrm{P}_{3}(\mathrm{t}), \\
\mathrm{P}_{4}^{\prime}(\mathrm{t})= & \delta \cdot \mathrm{P}_{3}(\mathrm{t})-\mu \cdot \mathrm{P}_{4}(\mathrm{t}), \\
\mathrm{P}_{5}^{\prime}(\mathrm{t})= & \lambda_{\mathrm{LC}}^{\mathrm{D}} \mathrm{P}_{1}(\mathrm{t})+\left(\lambda_{\mathrm{S}}+\lambda_{\mathrm{LC}}^{\mathrm{D}}\right) \cdot \mathrm{P}_{2}(\mathrm{t})+\left(\lambda_{\mathrm{S}}+\right. \\
& \left.+\lambda_{\mathrm{LC}}^{\mathrm{D}}\right) \cdot \mathrm{P}_{3}(\mathrm{t}) \cdot
\end{aligned}
$$

\section{EXPERIMENTAL RESULTS}

When modeling the probability of a dangerous failure LC or LC with MUTING function and presenting experimental results the following assumptions will be considered:

- The light curtain used has a rate of dangerous failures $\lambda_{L C}^{D}=9,6 \cdot 10^{-9} \mathrm{~h}^{-1}$ (safety light curtain (Sick - deTec4 Prime)).

- We use two types of additional sensors (to highlight their safety effect) better with the rate of failures $\quad \lambda_{S}=\lambda_{S 1}=$ $\lambda_{S 2}=4 \cdot 10^{-5} \mathrm{~h}^{-1}$ and less quality with failure rate $\lambda_{S}=\lambda_{S 1}=\lambda_{S 2}=9 \cdot 10^{-5} \mathrm{~h}^{-1}$.

- Detection of sensor failures is a matter of mutual comparison therefore a detectable failure of the sensor is one that is reflected by a change in the logic value at its output, and an undetectable failure is the one that does not occur by changing the logic value at its output. Given the generally acceptable symmetrical manifestation of the failure of the electronic elements, we assume the diagnostic coverage $c=0,5$.

- Object transition by light curtain at two different time intervals - short $t_{\max }=1 \mathrm{~min}$ and long $t_{\max }=8$ hod.

- $\quad$ Allowed sensor time discrepancy $t_{\text {disc }}=1 \mathrm{~s}$.

- Mean down time $M D T=1 \mathrm{~h}$ (it is only one sensor replacement after detecting its failure, assuming the sensor is part of the stock).

Fig. 6 shows the time course of probability of a LC and LC failure with MUTING. The graph in Fig. 6a) captures the time in the range of $0 \mathrm{~h}-180000 \mathrm{~h}$ (approximately 20 years, the commonly considered life of the systems). The graph in Fig. 6b) shows a more detailed view of curves 3, 4 and 5, which in Fig. 6a) partially coincide. Curves 1-4 illustrate the probability of dangerous failure of a LC with MUTING function, curve 5 is shown for comparison, and represents the probability of dangerous failure of a LC without MUTING function. The curves are based on the CTMC in Fig. 4 and Fig. 5 and model parameters for each run are shown in Table I.

Table I. The curve parameters show in Fig. 6

\begin{tabular}{|c|l|c|c|}
\hline curve & model & $\lambda_{S}\left[\mathrm{~h}^{-1}\right]$ & $t_{\max }[\mathrm{h}]$ \\
\hline $\mathbf{1}$ & Fig. 5 & $9.10^{-5}$ & 8 \\
\hline 2 & Fig. 5 & $4.10^{-5}$ & 8 \\
\hline 3 & Fig. 5 & $9.10^{-5}$ & $0,0167(1 \mathrm{~min})$ \\
\hline 4 & Fig. 5 & $4.10^{-5}$ & $0,0167(1 \mathrm{~min})$ \\
\hline $\mathbf{5}$ & Fig. 4 & - & - \\
\hline \multicolumn{2}{|c|}{$\lambda_{L C}^{D}=9,6.10^{-9} \mathrm{~h}^{-1} ; t_{\text {disc }}=1 s ; M D T=1 \mathrm{~h}$} \\
\hline
\end{tabular}



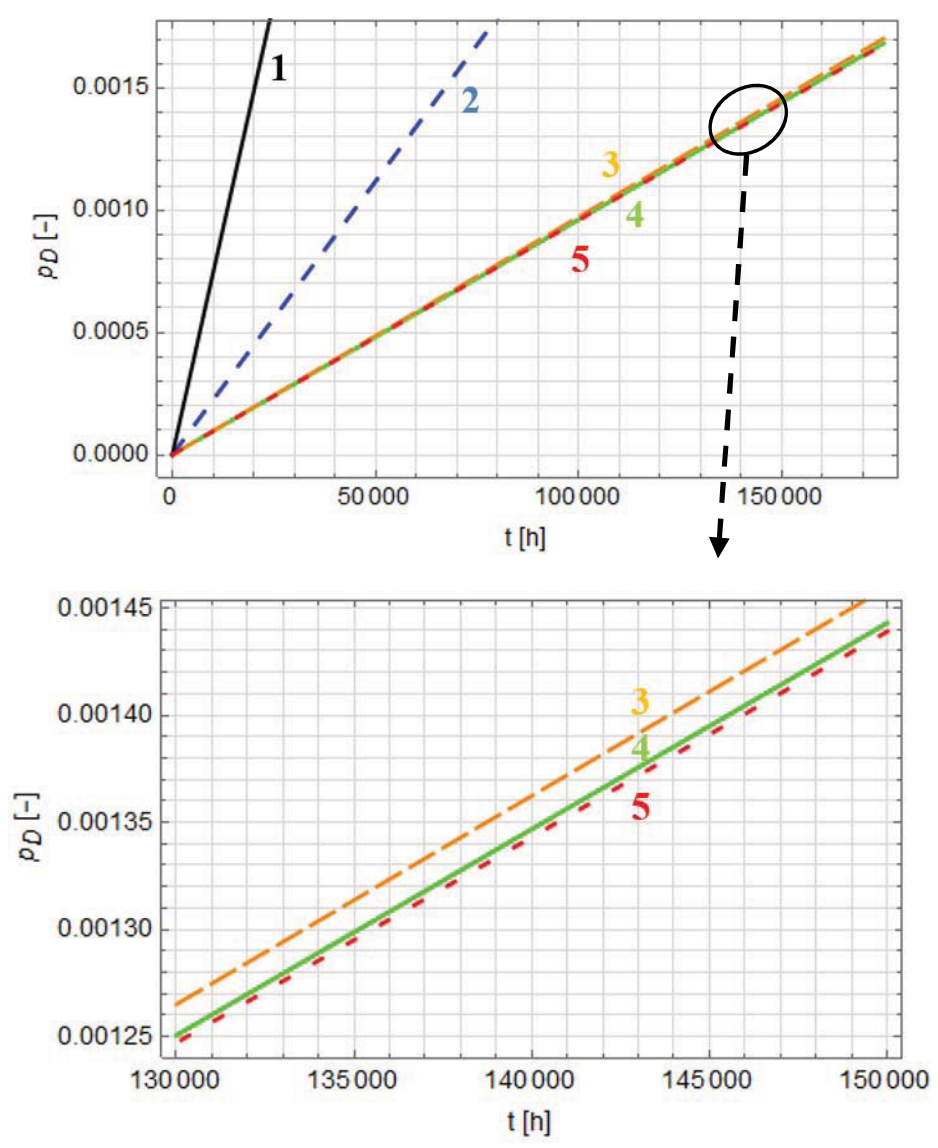

Fig. 6. Probability dangerous failure of LC and LC with MUTING function

From the curves in Fig. 6 we can see that the probability of dangerous failure of a LC without MUTING function is the lowest one. It is caused by the fact that the remaining four curves are assembled for LC with MUTING function and therefore they use additional sensors that also affect the probability of a dangerous failure. However, it is important to analyze curves 1-4 as they perform the same function and indicate the effect of the rate failure of the additional sensor $\lambda_{S}$ and the effect of the length of the interval between two consecutive objects $t_{\max }$.

An analysis of the processes shows that a significant safety impact is to detect sensor failures. MUTING devices have only functional diagnostics (the evaluation algorithm does not have a test diagnostic at the application level), therefore the efficiency of failure detection depends on the change in the status that occurs when evaluating the presence of the object (parameter $t_{\max }$ significantly affects the effectiveness of functional diagnostics). This can also be seen from a comparison of the 1,3 and 2,4 . At the same rate of the sensor failures, the course with greater $t_{\max }$ (curve 1 and 2) has significantly higher probability of failure than a course with smaller $t_{\max }$ (curve 3 and 4). It is also possible to say that for a longer time $t_{\max }$ (less efficient diagnostics, less frequent to verify the functionality of the additional sensors), the influence of the rate of the failures of the additional sensors is more considerable (curves 1,2 ) than at lower time $t_{\max }$ (curves 3, 4).

\section{CONCLUSION}

Protected area monitoring using the light curtain is one of the frequently used safety functions. In many cases, such features are deployed on a routine basis without more detailed analysis of the impact of various factors on safety. The paper analyzes the impact of selected factors on function safety with the MUTING function with the light curtain. Safety factors have been selected so that the results of the contribution could be used in practical applications. When performing safety functions, the integrity of safety against systematic errors must be met, so it is appropriate to use the certified function blocks or firmware of the certified light curtain to evaluate the information with additional sensors. More complex view on the implementation of safety function with a light curtain would be gained by analysis of the influence of the parameters on other properties, especially on reliability of the implemented functions. That kind of analysis, however, is not part of the scope of this paper.

\section{ACKNOWLEDGMENT}

This work has been supported by the Educational Grant Agency of the Slovak Republic (KEGA) Number 016ZU-4/2018: Modernization of teaching methods of management of industrial processes based on the concept of Industry $4.0(50 \%)$ and Number 008ŽU-4/2019: Modernization and expansion of educational possibilities in the field of safe controlling of industrial processes using the safety PLC (50\%). 


\section{REFERENCES}

[1] EN IEC 61508, "Functional safety of electrical/ electronic/programmable electronic safety-related systems," 2010.

[2] J. Ždánsky, K. Rástočný, "Influence of Safety PLC Parameters to Response Time of Safety Functions," Proceedings of International Conference Applied Electronics, AE 2013, Pilsen, Czech Republic, Sep 10-12, p. 327-330, ISBN 978-80261-0166-6, ISSN 1803-7232.

[3] J. Hrbček, P. Božek, J. Svetlík, V. Šimák, M. Hruboš, D. Nemec, A. Janota, E. Bubeníková, "Control system for the haptic paddle used in mobile robotics," International Journal of Advanced Robotic Systems, Sage journals, Vol. 14, No. 5, 2017, p. 1 - 11, ISSN 1729-8814.

[4] D. Darvas, I. Majzik, EB. Vinuela, "Formal Verification of Safety PLC Based Control Software," 12th International Conference on Integrated Formal Methods, Reykjavik, Iceland, Jun 01-05, 2016, p. 508-522, ISBN 978-3-319-33693-0, ISSN 0302-9743.

[5] S. Biallas, V. Kamin, S. Kowalewski, B. Schlich, S. Sehestedt, S. Stattelmann, "Verification of Safety-Critical PLC Programs using Safety Automata," 14th Branch Meeting of Measurement and Automation Technology, Baden, Germany, p. 75-79, 2013, ISBN 978-3-18-092209-6, ISSN 0083-5560.

[6] K. Rástočný, A. Janota, J. Zahradník, "The use of UML for development of a railway interlocking system," Lecture Notes in Computer Science (including subseries Lecture Notes in Artificial Intelligence and Lecture Notes in Bioinformatics) Springer, Volume 3147, Pages 174-198, 2004, ISSN: 03029743.
[7] K. Rastocny, M. Franekova, I. Zolotová, et al., "Quantitative Assessment of Safety Integrity Level of Message Transmission between Safety-related Equipment," Computing and Informatics, Volume 33, Issue 2, Pages 343-368, 2014, ISSN 1335-9150.

[8] J. Ždánsky, K. Rástočný, J. Hrbček, "Influence of Architecture and Diagnostic to the Safety Integrity of SRECS Output Part," 20th International Conference on Applied Electronics, Pilsen, Czech Republic, 2015 September 8-9, p. 297-301, ISBN 978802610385-1.

[9] M. Franeková, K. Rástočný, "Safety Evaluation of Fail-safe Fieldbus in Safety Related Control System, Journal of Electrical Engineering," Volume: 61 Issue: 6 Pages: 350-356, 2010, ISSN: 1335-3632, DOI: 10.2478/v10187-010-0054-z.

[10] M. Rousand, "Reliability of Safety-Critical Systems, Theory and Applications," Published by John Wiley \& Sons, Hoboken, New Jersey, 2014, ISBN: 978-1-118-11272-4.

[11] D. Smith, "The Safety Critical System Handbook," Published by Elsevier, 2016, ISBN 978-0-12-805121-4.

[12] J. Durech, M. Franeková, P. Holečko, E. Bubeníková, "Modelling of security principles within car-to-car communications in modern cooperative inteligent transportation system," In: Advances in electrical and electronic engineering, 2016, ISSN 1336-1376, Vol. 14, No. 1, pp. 49-58.

[13] HM. Lu, S. Serikawa, "Design of Freely Configurable Safety Light Curtain Using Hemispherical Mirrors," IEEJ Transactions on Electrical and Electronic Engineering, DOI: 10.1002/tee.21928, 2013, Pages: S110-S111, USA.

[14] EN ISO 13855, "Safety of machinery.Positioning of safeguards with respect to the approach speeds of parts of the human body," 2010. 